\title{
TWO-SCALE THEORY OF EDGE STATE
}

\author{
A. Matulis \\ Semiconductor Physics Institute, Center for Physical Sciences and Technology, Sauletekio 3, LT-10222 Vilnius, Lithuania \\ E-mail: algirdas.matulis@ftmc.lt
}

Received 4 September 2016; revised 6 October 2016; accepted 21 December 2016

\begin{abstract}
The edge state is considered in the spectrum region where its branch splits from the bottom of a continuous conduction band. It is shown that in this region the electron wave function demonstrates two different scale behaviours: slow and fast, that enabled us to construct some simplified procedure for the analysis of the edge state. The slow wave function part obeys a simple Schrödinger equation the parameters of which are insensitive to the peculiarities of the electron dynamics, while the fast part that describes the details of electron behaviour in the primitive cell reveals itself only at the edge. The equation for this fast part was transformed into the boundary condition for the slow part equation. The proposed method is illustrated considering the simplest continuous model for a topological insulator and a tight binding model for graphene.
\end{abstract}

Keywords: continuous model, tight binding model, splitting point, fast-slow wave function parts

PACS: 3.65.Ge, 73.20.-r, 73.22.-f, 73.22.Pr

\section{Introduction}

The topological insulators [1] have been a focus of intense recent interest. The attention is mainly related to the protected edge or surface states appearing in the forbidden energy gap. These states lead to a conducting state of the insulator with properties unlike any other known in electronic structures. In addition to the fundamental interest in them, these states are predicted to have special properties that could be useful for applications ranging from spintronics to quantum computation. The topological insulator is closely related to the quantum Hall state that reveals itself in single layer 2D (two-dimensional) systems (like in graphene [2]) where the role of the external magnetic field is played by the internal one caused by the spinorbit interaction of electrons [3].

The most interesting and promising achievement is based on the topological symmetry, the ideas of which about the Berry phase [4], invariant Chern numbers [5, 6] and topological order [8, 8] enabled one to make the classification of new topological phases in solid state physics and predict the appearance of topologically protected helical edge states. The edge state problem is always more complicated than the bulk state one due to breaking of the translation symmetry. That is why very often the numerical calculations have to be used. Meanwhile the solutions of simplified model problems by analytical means always provide useful information for understanding of more complicated systems [9, 10]. See also [11] where the properties of interacting 1D topological insulators are interpreted in terms of Green's functions.

The aim of this paper is to demonstrate that a useful analytical consideration of the spectrum branch of the edge state is possible close to the point where it splits from the continuous band making use of a small parameter, namely, the energy deviation from the splitting point as compared with the forbidden energy gap. We show that in this region it is possible to construct the electron wave function of the edge state of two different scale parts that reminds of the adiabatic approach in the molecular vibration theory where two-scale evolution in time is used. We succeeded to reformulate the fast wave function part equation into the boundary condition for the slow part one that actually determines the conditions of the edge state appearance. We demonstrate this idea using two toy models: a continuous version [12] of the Bernevig [13] model of a $2 \mathrm{D}$ topological insulator and the discrete TBM (tight binding model) Haldane model [3] of graphene that 
includes spin-orbit interaction and electron hopping to the next nearest neighbours.

The paper is organized as follows. Section 2 is devoted to a continuous topological insulator model. So, in Subsection 2.1 the half plane problem is settled and in Appendix $\mathrm{A}$ it is reformulated as an equation for a single wave function component. In Subsection 2.2 the two-scale behaviour of the wave function is revealed and the proper matching procedure is proposed. In Subsection 2.3 the behaviour of the edge state close to the point where it splits from the conduction band is discussed and the results are presented. In Section 3 and Appendix B the same procedure for the description of graphene in the TBM approach is presented. In the last Section 4 our conclusions are given.

\section{Topological insulator}

We demonstrate the idea of the proposed method considering the simplest continuous model of a $2 \mathrm{D}$ topological insulator that according to [12] is characterized by the Hamiltonian ( $4 \times 4$ matrix)

$$
\hat{H}=\left(\begin{array}{cc}
\hat{\mathcal{H}} & 0 \\
0 & \hat{\mathcal{H}}^{*}
\end{array}\right)
$$

composed of two $2 \times 2$ blocks:

$$
\hat{\mathcal{H}}=\left(\begin{array}{cc}
M+B \nabla^{2} & -\mathrm{i} A(\partial / \partial x-\mathrm{i} \partial / \partial y) \\
-\mathrm{i} A(\partial / \partial x+\mathrm{i} \partial / \partial y) & -M-B \nabla^{2}
\end{array}\right) .
$$

Because of the absence of off-diagonal blocks in Hamiltonian (1), one can analyze states for each block separately. We consider the upper block.

Denoting the wave function as

$$
\Psi=\left(\begin{array}{l}
u \\
v
\end{array}\right),
$$

scaling coordinates $\{x, y\} \rightarrow(B / A)\{x, y\}$, replacing the constant $M \rightarrow\left(A^{2} / B\right) M$ and measuring the electron energy $E$ in $A^{2} / \mathrm{B}$ units, we have the following eigenvalue problem composed of two differential equations for the wave function components:

$$
\begin{aligned}
& \left(M+\nabla^{2}\right) u-\mathrm{i}(\partial / x-\mathrm{i} \partial / \partial y) v=E u, \\
& -\mathrm{i}(\partial / \partial x+\mathrm{i} \partial / \partial y) u-\left(M+\nabla^{2}\right) v=E v,
\end{aligned}
$$

controlled by a single dimensionless constant $M$.

Assuming the exponential type wave function

$$
\left(\begin{array}{l}
u(x, y) \\
v(x, y)
\end{array}\right)=\mathrm{e}^{\mathrm{i}(k x+q y)}\left(\begin{array}{l}
u \\
v
\end{array}\right)
$$

and inserting it into Eqs. (4), we obtain the dispersion relation

$$
E^{2}=\mathcal{E}^{2}(p)=p^{4}-(2 M-1) p^{2}+M^{2}
$$

in the case of electron moving in the infinite plane, where

$$
p^{2}=k^{2}+q^{2} .
$$

The corresponding wave function components are

$$
\left(\begin{array}{l}
u \\
v
\end{array}\right)=\left(\begin{array}{c}
E+M-p^{2} \\
k+\mathrm{i} q
\end{array}\right) \text {. }
$$

The spectrum has the cylindric symmetry. Its two branches are shown in Fig. 1 (panel (a)) by solid curves as functions of the absolute value of the electron momentum $p$ (7). The projection of this spectrum onto the $k=0$ plane is shown in the panel (b), where the continuous conduction and valence bands are indicated by shadowed areas. In the case of $M>1 / 2$ the upper (lower) branch has the minimum (maximum) on the ring with the radius $p_{0}=\sqrt{M-1 / 2}$ denoted by points $K(L)$ that are the points of our interest.
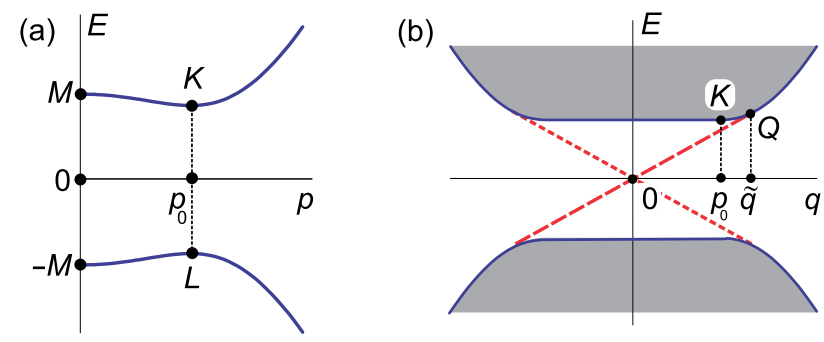

Fig. 1. Spectrum (coloured online) according to Eq. (6a) and its projection onto the $k=0$ plane (b).

\subsection{Half-plane problem}

Now we consider the eigenvalue problem on the halfplane $0 \leq x<\infty,-\infty<y<\infty$, where the electron motion is described by the same Eqs. (4) with the boundary conditions $u(0, y)=v(0, y)=0$ added. This system is invariant in respect to the translation along the $y$ axis. That is why the electron momentum component $q$ is a good quantum number, and the wave function can be presented as

$$
\left(\begin{array}{l}
u(x, y) \\
v(x, y)
\end{array}\right)=\mathrm{e}^{\mathrm{i} q y}\left(\begin{array}{l}
u(x) \\
v(x)
\end{array}\right)
$$

Inserting it into (4) we transform those equations into the following set of two ordinary differential equations: 


$$
\begin{aligned}
& \left(E-M+q^{2}-\frac{\mathrm{d}^{2}}{\mathrm{~d} x^{2}}\right) u=-\mathrm{i}\left(\frac{\mathrm{d}}{\mathrm{d} x}+q\right) v, \\
& \left(E+M-q^{2}+\frac{\mathrm{d}^{2}}{\mathrm{~d} x^{2}}\right) v=-\mathrm{i}\left(\frac{\mathrm{d}}{\mathrm{d} x}-q\right) u,
\end{aligned}
$$

with the boundary conditions

$$
\begin{aligned}
& u(0)=0, \\
& v(0)=0 .
\end{aligned}
$$

We shall present all results and plots as functions of that quantum number $q$ : the electron momentum along the edge. The continuous bands are actually described by the same dispersion relation (6) as in the case of infinite plane, only having twice smaller density of states, and, consequently, they coincide with the shadowed regions shown in the panel (b) of Fig. 1. Minimizing that dispersion relation in respect to the perpendicular component of the electron momentum $k$ we obtain the following minimum (maximum) of these upper (lower) energy bands:

$$
\varepsilon_{0}^{2}(q)= \begin{cases}M-1 / 4, & q^{2}<p_{0}^{2} \\ \varepsilon^{2}(q), & q^{2}>p_{0}^{2}\end{cases}
$$

According to [12] two edge state branches appear in the forbidden energy gap. We restrict ourselves to the one shown by a dashed (red) curve which splits from the upper continuous energy band close to the previously mentioned point $K$. The other edge state (indicated by a dotted curve) follows from the lower block of Hamiltonian (1), or by a simple replacement $q \rightarrow-q$ in the final result.

\subsection{Two scales of the wave function}

Constructing the approximate description of the edge state close to the point $K$ it is more convenient to use a single equation instead of a set of two Eqs. (10). This equation for the component $u$ (see the derivation in Appendix A) reads

$$
\frac{\mathrm{d}^{4} u}{\mathrm{~d} x^{4}}-2 R \frac{\mathrm{d}^{2} u}{\mathrm{~d} x^{2}}+w^{2} u=0
$$

where

$$
\begin{aligned}
& R=q^{2}-M+1 / 2, \\
& w^{2}=\mathcal{E}^{2}(q)-E^{2},
\end{aligned}
$$

and the following boundary conditions have to be added:

$$
\begin{aligned}
& u(0)=0, \\
& u^{\prime \prime \prime}(0)-q u^{\prime \prime}(0)-\left(E+q^{2}-M+1\right) u^{\prime}(0)=0 .
\end{aligned}
$$

As already mentioned, our main interest is the small region around the point $K$ where the edge state splits from the minimum of the upper continuous band. This minimum is given by the function $\mathcal{E}(q)$ what makes the coefficient $w$ small (see Eq. (15)). The smallness of the last term in Eq. (13) leads to two characteristic scales of the wave function $u(x)$ behaviour, which enables us to construct the component $u(x)$ by some approximate procedure.

We start the consideration of that approximate procedure neglecting the small last term in Eq. (13) and rewriting it as

$$
\frac{\mathrm{d}^{2}}{\mathrm{~d} x^{2}}\left(\frac{\mathrm{d}^{2}}{\mathrm{~d} x^{2}}-2 R\right) u(x)=0 .
$$

The general solution with an arbitrary chosen normalization factor reads

$$
u_{\mathrm{f}}(x)=-\mathrm{e}^{-\kappa x}+A \mathrm{e}^{\kappa x}+B+C x,
$$

where

$$
\kappa=\sqrt{2 R} .
$$

The second term in Eq. (18) has to be omitted as we are looking for the edge state the wave function of which descends receding from the edge. Thus $A=0$. In order to satisfy the boundary condition (16a) we assume that $B=1$. The last constant $C$ follows from the second boundary condition $(16 \mathrm{~b})$ and it reads

$$
C=\kappa\left[\frac{\kappa(\kappa+q)}{E+q^{2}-M+1}-1\right] \text {. }
$$

The constructed fast part of the wave function

$$
u_{\mathrm{f}}(x)=-\mathrm{e}^{-\kappa x}+1+C x
$$

is correct close to the origin $(x=0)$, where its coordinate dependence is strong, and, consequently, the neglected term in (13) is small as compared with two other terms with derivatives. But in the asymptotic region $(x \rightarrow \infty)$ the function is not correct due to a slowly growing last term in formula (21). In this region some other approximation has to be used for the calculation of the slow wave function part. Here we neglect the first term with the highest derivative in Eq. (13) rewriting it as

$$
2 R \frac{\mathrm{d}^{2} u}{\mathrm{~d} x^{2}}-w^{2} u=0
$$


The solution suitable for us (namely, descending wave function when receding from the edge) reads

$$
u_{s}(x)=D \mathrm{e}^{-\gamma x},
$$

where

$$
\gamma=\frac{w}{\sqrt{2 R}} .
$$

Both wave function parts (fast and slow) have to be matched together. As the slow part obeys the differential equation of the second order it is sufficient to match the functions and their first derivatives. The idea of this matching is shown in Fig. 2. The upper and lower (red and blue online) curves correspond to the slow $u_{\mathrm{s}}$ and fast $u_{\mathrm{f}}$ wave function parts calculated above. The solid parts of these curves correspond to the regions where the functions are sufficiently accurate. We assume that these parts have to be matched at some intermediate point $x_{0}$.

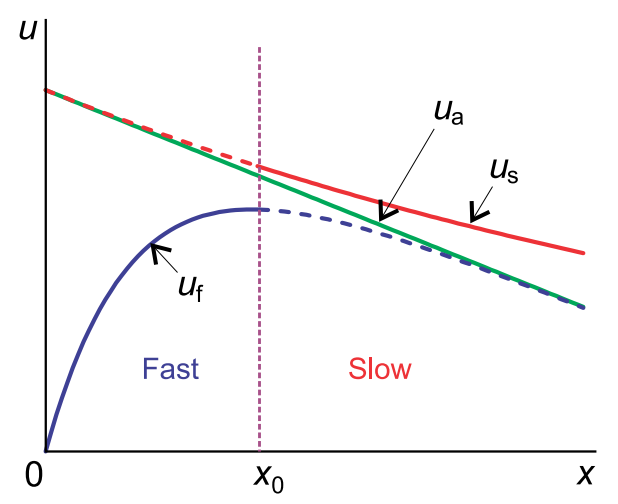

Fig. 2. Matching of the fast and slow wave function parts (coloured online).

Due to the smallness of parameter (15), and, as a consequence, the smallness of parameter $\gamma$ as well (see formula (24)) it is possible to choose the matching point satisfying the following conditions:

$$
\kappa x_{0} \gg 1, \quad \gamma x_{0} \ll 1 .
$$

Because of the first condition the exponent of the fast function (21) may be neglected taking into account only its asymptotic part

$$
u_{\mathrm{a}}(x)=1+C x,
$$

shown in Fig. 2 by a straight solid (green) line. It represents the fast part of the wave function quite close to the point $x_{0}$ where we are going to perform the matching. The second condition in (25) enables us to simplify the slow function (23) as well. Name- ly, we can change the matching point to $x_{0}=0$. So, matching the functions and their derivatives we get

$$
\left.\frac{u_{\mathrm{a}}^{\prime}}{u_{\mathrm{a}}}\right|_{x=0}=C=\left.\frac{u_{\mathrm{s}}^{\prime}}{u_{\mathrm{s}}}\right|_{x=0}=-\gamma,
$$

or

$$
w=2 R\left[1-\frac{\kappa(\kappa+q)}{E+q^{2}-M+1}\right] .
$$

This is the final algebraic equation that has to be solved together with Eq. (15) for the edge state energy definition. Assuming $w=0$ one can easily define the coordinates of point $Q$ (see panel (b) in Fig. 1) where the edge state energy branch enters the continuous band:

$$
E_{0}=\widetilde{q}=\sqrt{M} .
$$

\subsection{Expansion close to $Q$ point}

We have to remember that the two scales in the wave function behaviour, on which the consideration presented in Subsection 2.2 was based, appeared due to the closeness of the edge state energy branch to the point $Q$. That is why there is no need to solve Eq. (28) exactly, and we introduce deviations of energy and momentum from the above point assuming that they are small:

$$
E=E_{0}+\varepsilon, \quad q=\tilde{q}+g, \quad \varepsilon, g \ll 1 .
$$

In order to obtain the edge state energy $\varepsilon$ within the accuracy of $g^{2}$ terms we have to square Eq. (28) and replace its left side by Eq. (15) restricted to the following expansion:

$$
w^{2}=2 \sqrt{M}(g-\varepsilon)+(1+4 M) g^{2}-\varepsilon^{2} .
$$

Meanwhile, due to the proper choice of zero term (29) the expression in brackets in the right hand side of Eq. (28) has no zero order terms. Consequently, the choice of the first order terms in the brackets and the zero order approximation of the prefactor, namely, the replacement of (28) by the equation

$$
w=-\left[\frac{g-\varepsilon}{1+\sqrt{M}}+2 \sqrt{M} g\right]
$$

is quite sufficient for our purpose. Now comparing this equation with Eq. (31) we obtain the following equation: 


$$
\begin{aligned}
& \sqrt{2 \sqrt{M}(g-\varepsilon)+(1+4 M) g^{2}-\varepsilon^{2}} \\
& =-\left[\frac{g-\varepsilon}{1+\sqrt{M}}+2 \sqrt{M} g\right] .
\end{aligned}
$$

The sign of the radical should be positive due to the positiveness of the decrement $\gamma$ in the case of edge state. This equation can be satisfied by the function

$$
\varepsilon=g
$$

Indeed, inserting this function into Eq. (33) we obtain the condition

$$
\sqrt{4 M g^{2}}=-2 \sqrt{M} g
$$

that is satisfied identically in the case of negative $g$ values. This result is shown in the panel (b) of Fig. 1 by a dashed (red online) line and coincides with the one obtained in [12] by means of a more complicated exact consideration.

It is remarkable that in this consideration the condition $M>1 / 2$ was not actually used. Consequently, the existence of edge states is not related to the presence of $K(L)$ points in the spectrum. Thus, the $Q$ point (33) and the edge state branch (34) preserve themselves till $M=0$ when the energy gap closes.

It is worth drawing attention to the possibility of peculiar interpretation of boundary condition (27). Indeed, close to the point $Q$ on the edge state branch $(\varepsilon=g)$ the boundary condition turns into $u_{\mathrm{s}}^{\prime} / u_{\mathrm{s}}=2 \sqrt{M} g$ that can be taken into account by means of formal extending symmetrically the solution to negative $x$ values and adding the potential $4 g \sqrt{M} \delta(x)$ to Eq. (22) for the slow wave function part. In the $1 \mathrm{D}$ case and $g<0$ such negative Dirac type local potential always leads to a single bound state corresponding to the above considered edge state. This simple consideration gives some physical meaning to the procedure indicating that close to the splitting point the exponent type wave function of the edge state satisfies a simple Schrödinger equation for a free electron, while the sophisticated dynamics of the electron provides the boundary condition (equivalent to some local potential).

\section{Graphene}

Now we are going to show that the method proposed in the previous section has a more wide application considering the discrete model of graphene, namely, TBM (tight binding model) when the electron tunnelling to the next neighbouring atoms and the spin-orbit interaction are taken into account.
We consider the motion of an electron in the hexagonal lattice shown in Fig. 3. According to [3] the electron motion is described by the following TBM equations:

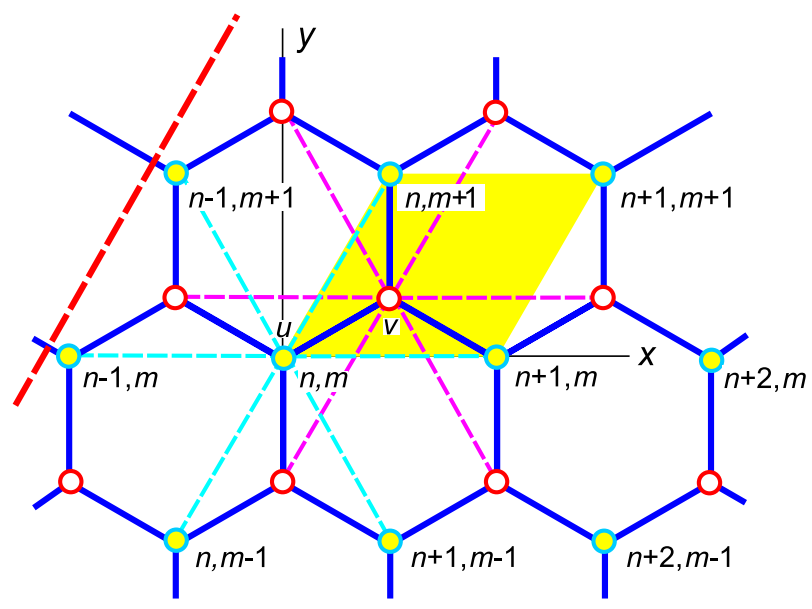

Fig. 3. Graphene lattice (coloured online).

$$
\begin{aligned}
& E u_{n, m}=\left(v_{n, m}+v_{n-1, m}+v_{n, m-1}\right) \\
& +\mathrm{i} t\left(u_{n, m+1}-u_{n-1, m+1}+u_{n-1, m}\right. \\
& \left.-u_{n, m-1}+u_{n+1, m-1}-u_{n+1, m}\right), \\
& E v_{n, m}=\left(u_{n, m}+u_{n+1, m}+u_{n, m+1}\right) \\
& +\mathrm{i} t\left(v_{n, m+1}-v_{n-1, m+1}+v_{n-1, m}\right. \\
& \left.-v_{n, m-1}+v_{n+1, m-1}-v_{n+1, m}\right) .
\end{aligned}
$$

In order to simplify the notations we use the dimensionless variables when the energy is measured in the tunnelling to the nearest neighbour amplitude units. The coordinates are measured in units of distance to the next neighbouring sites indicated by thin dashed (violet and light blue) lines. The symbol $t$ means the ratio of two tunnelling amplitudes: to the next neighbours and to the nearest ones. It was shown experimentally in [14] and theoretically in [15, 16] that this ratio in graphene is of order $10^{-6}$. It is negligible and, thus, the effects related with the quantum spin Hall insulator can reveal themselves in graphene at very low temperatures only. Nevertheless, following [3] we shall use that Hamiltonian with the exaggerated constant $t$ (say, $t=0.1$ ) as a toy model for the illustration of the proposed technique.

In this model the electron motion is actually described by four amplitudes. The last two components differ from the two ones under the consideration by different spin. They satisfy the equations similar 
to Eqs. (36) but with an opposite sign of imaginary terms. As there is no interaction between these amplitude pairs they can be considered separately, and we shall choose the first pair of them.

We consider the zigzag edge. That is why we assume that the plane shown in Fig. 3 is cut along the thick dashed (red) line corresponding to the index $n=1$. In order to use the same Eqs. (36) for the amplitudes $u_{0, m}$ and $v_{0, m}$ at the edge we assume the following boundary conditions:

$$
u_{-1, m}=v_{-1, m}=0 \text {. }
$$

Following the procedure presented in the previous section and taking into account the translation symmetry along the edge we transform the problem into $1 \mathrm{D}$ one assuming the following wave function:

$$
\left(\begin{array}{l}
u_{n, m} \\
v_{n, m}
\end{array}\right)=\mathrm{e}^{\mathrm{i} q(m+n / 2)}\left(\begin{array}{c}
u_{n} \\
\mathrm{e}^{\mathrm{i} q / 2} v_{n}
\end{array}\right) .
$$

It enables us to write down the following set of two $1 \mathrm{D}$ equations,

$$
\begin{aligned}
& (E+\tau \lambda) u_{n}-\tau\left(u_{n-1}+u_{n+1}\right)=\lambda v_{n}+v_{n-1}, \\
& (E-\tau \lambda) v_{n}+\tau\left(v_{n-1}+v_{n+1}\right)=\lambda v_{n}+u_{n+1} .
\end{aligned}
$$

Here

$$
\lambda \equiv \lambda(q)=2 \cos (q / 2), \quad \tau \equiv \tau(q)=2 t \sin (q / 2) .
$$

The applied substitution transforms the boundary condition (37) to

$$
\begin{aligned}
& u_{-1}=0, \\
& v_{-1}=0 .
\end{aligned}
$$

We see that the motion of the electron in this model is controlled by two parameters $\lambda$ and $\tau$ that depend on the momentum component along the edge $q$ and the spin-orbit interaction constant $t$ (as it was in the continuous model of the topological insulator considered in the previous section).

Now inserting the exponential type amplitudes

$$
u_{n}, v_{n} \sim \mathrm{e}^{\mathrm{i} k n}
$$

into Eqs. (39) we obtain the spectrum for the electron moving in the infinite plane

$$
E^{2}=16 \tau^{2} \sin ^{4}(k / 2)-4 R \sin ^{2}(k / 2)+\mathcal{E}^{2}(q),
$$

where

$$
\begin{aligned}
& R=-\lambda+2 \tau^{2}(\lambda-2), \\
& \mathcal{E}^{2}(q)=(1+\lambda)^{2}+\tau^{2}(2-\lambda)^{2} .
\end{aligned}
$$

Its upper band is indicated in Fig. 4 by solid (blue) curves as functions of the electron momentum component parallel to the edge $q$ for various $k$ values. The lower band can be obtained right from it just inverting the energy axis.

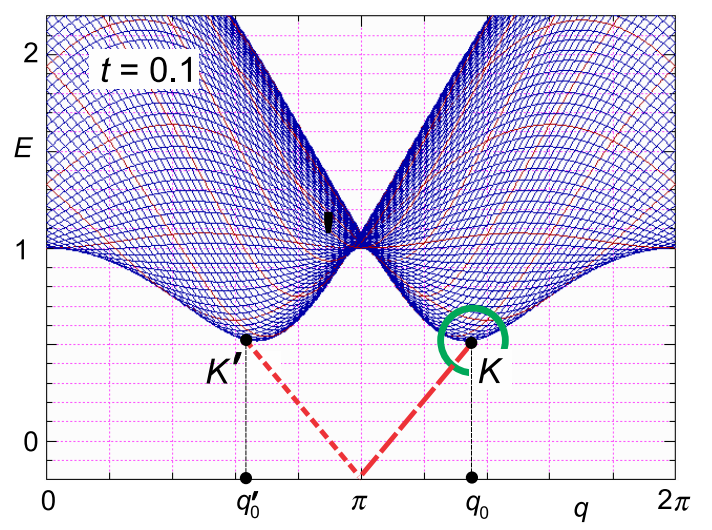

Fig. 4. Spectrum (coloured online) according to Eq. (43).

It follows from Eq. (43) that the minimum of the upper band at given $q$ is achieved when $k=0$. This lower edge of the continuous spectrum is given by function $\mathcal{E}(q)$. It has the minimum at points $K$ and $K$ ', where

$$
q_{0}=4 \pi / 3, \quad q_{0}^{\prime}=2 \pi / 3, \quad \lambda_{0}=-1, \quad E_{0}=3 \sqrt{3} t .
$$

It is remarkable that the position of these minima does not depend on the parameter $t$.

\subsection{Half-plane problem}

In the case of half-plane the continuous spectrum remains the same, but the additional edge state branches appear splitting from the continuous band close to the specific $K$ and $K$ ' points. Schematically these edge state branches are shown by the dashed and dotted (red) curves in Fig. 4. Our main interest is the dashed curve, namely, the edge state in the vicinity of the right splitting point $K$, indicated by a small (green) circle.

It follows from Eqs. (45) and (39b) that $v_{n}=0$ at the point $K$. That is why one can expect that in a small region close to that point the amplitude $v_{n}$ remains small. Thus, it is worth to transform Eqs. (39) into a single equation for the large amplitude $u_{n}$, like it was done in Section 2. The details of this transformation are given in Appendix B, and the equation with boundary conditions reads 


$$
\begin{aligned}
& \tau^{2}\left(u_{n+2}-4 u_{n+1}+6 u_{n}-4 u_{n-1}+u_{n-2}\right) \\
& -R\left(u_{n+1}-2 u_{n}+u_{n-1}\right)+w^{2} u_{n}=0 \\
& u_{-1}=0 \\
& \tau^{2}\left(u_{1}-3 u_{0}+3 u_{-1}-u_{-2}\right) \\
& -F\left(u_{0}-2 u_{-1}+u_{-2}\right)+G\left(u_{0}-u_{-1}\right)=0
\end{aligned}
$$

where

$$
\begin{aligned}
& w^{2}=\mathcal{E}^{2}(q)-E^{2}, \\
& F=\tau^{2}(1+\lambda), \\
& G=\lambda+(2-\lambda) \tau^{2}-\tau E .
\end{aligned}
$$

Note all terms in the parentheses of Eqs. (46) are the analogues of derivatives.

\subsection{Two scales of the wave function}

Eq. (46a) for the description of the edge state in graphene includes the same small parameter $w$ as in the previous section. That is why we shall follow the same procedure distinguishing fast and slow regions of the wave function behaviour. First we omit the small last term in Eq. (46a) and solve the following truncated equation for the fast wave function part:

$$
\begin{aligned}
& \tau^{2}\left(u_{n+2}-4 u_{n+1}+6 u_{n}-4 u_{n-1}+u_{n-2}\right) \\
& -R\left(u_{n+1}-2 u_{n}+u_{n-1}\right)=0 .
\end{aligned}
$$

The solution of this difference equation can be constructed using the analogy with the differential equation, because the terms in parenthesis of this equation are the analogues of the forth and second derivatives in Eq. (17). It can be easily checked that the general solution can be chosen as

$$
u_{n}^{(f)}=1-\mathrm{e}^{-\kappa(n+1)}+\beta(n+1)
$$

with the decrement $\kappa$ satisfying the following truncated dispersion relation:

$$
4 \tau^{2} \sinh ^{2}(\kappa / 2)-R=0 .
$$

Constructing that solution we took into account the boundary condition (46b) and excluded the growing exponent.

Satisfying the second boundary condition (46c) we obtain one more equation,

$$
\begin{aligned}
& 2 \sinh (\kappa / 2)\left\{\mathrm{e}^{-\kappa / 2}\left[4 \tau^{2} \sinh ^{2}(\kappa / 2)+G\right]-2 F \sinh (\kappa / 2)\right\} \\
& +\beta G=0
\end{aligned}
$$

which together with Eq. (50) enables us to obtain the expression for the last constant in the fast part of the wave function (49), namely,

$$
\beta=-\frac{\sqrt{R}}{\tau G} W,
$$

where

$$
W=\mathrm{e}^{-\kappa / 2}(R+G)-\tau(1+\lambda) \sqrt{R}
$$

Looking for the slow wave function part we neglect the first parentheses in Eq. (46a) that corresponds to the derivative of the highest order, and rewrite it as follows:

$$
R\left(u_{n+1}-2 u_{n}+u_{n-1}\right)-w^{2} u_{n}=0
$$

The proper solution reads

$$
u_{n}^{(s)}=u_{0}^{(s)} \mathrm{e}^{-\gamma n}
$$

where the decrement $\gamma$ satisfies the following equation:

$$
4 \sinh ^{2}(\gamma / 2) \approx \gamma^{2}=\frac{w^{2}}{R} \ll 1 .
$$

Due to the smallness of the decrement $\gamma$ the replacement of sinus hyperbolic by its argument is quite reasonable.

The matching procedure is actually the same as that one in the case of differential equation (13). So, due to the inequality $\kappa \gg \gamma$ we choose such matching point $n_{0}$ that satisfies the following inequalities:

$$
\kappa n_{0} \gg 1, \quad \gamma n_{0} \ll 1 .
$$

Due to the first of them one can neglect the exponent in formula (49) and replace the fast wave function part (49) by its asymptote

$$
u_{\mathrm{n}}^{(\mathrm{a})}=1+\beta(n+1)
$$

that is adequate to the exact solution close to the matching point. Due to the second inequality it is possible to shift the matching point to the origin choosing $n_{0}=0$.

The difference equation (54) for the slow wave function part is the analogue of the differential 
equation with the second order derivative. That is why we need two boundary conditions for matching the slow wave function part (55) to the asymptotic formula (58) of the fast one. For instance, these two function parts can be equated at two neighbouring points, say, at $n_{0}=0$ and $n^{\prime}=-1$, which gives the following set of two equations:

$$
\begin{aligned}
& u_{0}^{(\mathrm{a})} \equiv 1+\beta=u_{0}^{(\mathrm{s})}, \\
& u_{-1}^{(\mathrm{a})} \equiv 1=u_{-1}^{(\mathrm{s})}=u_{0}^{(\mathrm{s})} \mathrm{e}^{\gamma} \approx(1+\beta)(1+\gamma),
\end{aligned}
$$

and

$$
\beta=-\gamma
$$

within the accuracy of linear term. Now taking Eqs. (56) and (52) into account we obtain the final equation

$$
w=\frac{R}{\tau G} W
$$

for the edge state energy calculation, similar to Eq. (28) that we used in the case of the continuous model for the topological insulator. This equation has to be considered together with definition (47a).

The point $Q$ where the edge state branch splits from the conduction band follows from the above equation when $w=0$ and $E=\mathcal{E}(\widetilde{q})$. For instance, in the case of $t=0.1$ the numerical solution of the above algebraic equation gives $\widetilde{q} / 2 \pi \approx 0.698$, and $E_{0} \equiv \mathcal{E}(\widetilde{q}) \approx 0.541$. This point does not coincide with the point $K$ that is located at $q=q_{0}=4 \pi / 3$ as shown in Fig. 5.

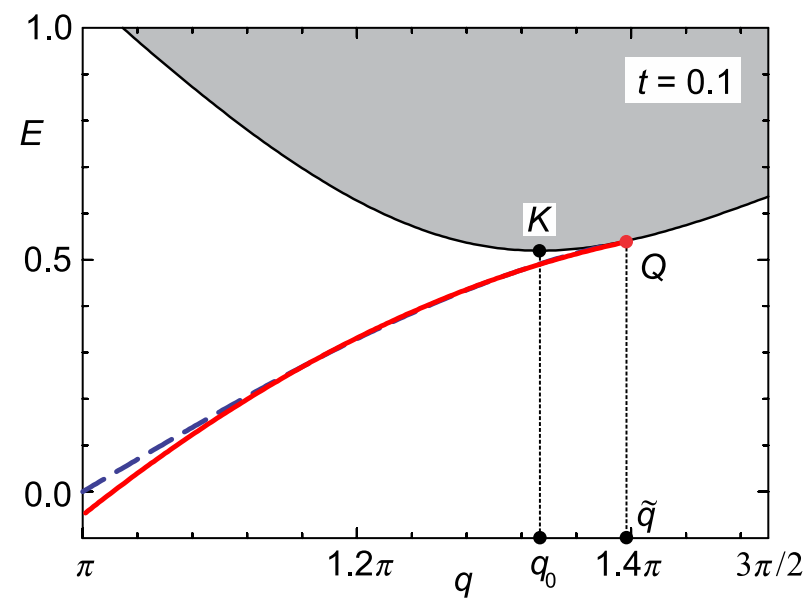

Fig. 5. Edge state branch: solid (red online) curve shows our result according to Eq. (66); dashed (blue online) curve is the solution of the edge problem obtained by the numerical diagonalization of the Hamiltonian corresponding to Eqs. (39); thick (black) curve is the bottom of the continuous band.

\subsection{Expansion close to $Q$ point}

The next step is evident. We expand the energy of electron and its momentum along the edge into a series of small deviations from the $Q$ point according to Eqs. (30). Following the consideration presented in Subsection 2.3 it is easy to reveal that in order to obtain the edge state energy within the accuracy of quadratic $g^{2}$ terms it is sufficient to replace Eqs. (44b) and (53) by the following truncated expansions:

$$
\begin{aligned}
& \mathcal{E}(q)=E_{0}+a g+b g^{2}, \\
& W=c g+d \varepsilon
\end{aligned}
$$

Here $a, b, c$ and $d$ are just the trivial coefficients in the Taylor series of the above parameters. Using Eqs. (47a) and (62a) we obtain

$$
w=\sqrt{2 E_{0}} \sqrt{(a+b g) g-\varepsilon},
$$

and inserting it into Eq. (61) together with the expansion $(62 \mathrm{~b})$ we arrive at the final simplified equation

$$
\sqrt{(a+b g) g-\varepsilon}=B(c g+d \varepsilon),
$$

where the coefficient

$$
B=\frac{R}{\tau G \sqrt{2 E_{0}}}
$$

is calculated at the splitting point $Q$ where $q=\widetilde{q}$.

Squaring Eq. (64) and iterating the small quadratic term $\varepsilon^{2}$ we obtain the solution

$$
\varepsilon=a g+\left[b-B^{2}(c+a d)^{2}\right] g^{2}
$$

that is valid for negative $g$ values and ensures the positiveness of the radical in Eq. (64).

This solution is illustrated by Fig. 5 where the edge state branch (66) is shown by the (red) solid curve in the case of $t=0.1$ when

$$
\begin{aligned}
& a \approx 1.260, \quad b \approx 15.84, \quad c \approx 1.101, \\
& d \approx-0.0257, \quad B \approx-5.054 .
\end{aligned}
$$

For comparison the (blue) dashed curve shows the exact solution of the edge problem obtained by the numerical diagonalization of the Hamiltonian corresponding to Eqs. (39). Good coincidence of these two curves confirms the adequacy of the proposed method that can be useful in a quite large region of the energy gap. When the parameter $t$ goes to zero, 
the energy gap closes and the edge state branches transform themselves into the horizontal lines inherent in the zig-zag edge state in graphene with the tunnelling to the nearest neighbours exclusively.

The procedure applied to the TBM problem has some simple physical meaning as well. Indeed, Eq. (54) resembles the eigenvalue problem for the free electron moving in the 1D lattice (in chain) tunnelling to the neighbouring sites with some tunnelling amplitude $R$, where according to Eq. (47a) $w^{2}$ represents the eigenvalue, namely, the electron energy counted from the bottom of the conduction band. In the case of $n=0$ this equation reads

$$
-w^{2} u_{0}^{(\mathrm{s})}=-R\left[\left(u_{1}^{(\mathrm{s})}-u_{0}^{(\mathrm{s})}\right)-\left(u_{0}^{(\mathrm{s})}-u_{-1}^{(\mathrm{s})}\right)\right] .
$$

Using solution (55) and condition (60) it can be easily transformed into the form

$$
-R\left(u_{1}-u_{0}\right)+R \beta u_{0}=-w^{2} u_{0}^{(s)}
$$

that can be interpreted as the equation for the amplitude $u_{0}$ at the edge of the half-infinite chain with the additional local potential $R \beta$. It follows from Eqs. (52), (62b) and (66) that close to the splitting point $Q$ the parameter $\beta$ is proportional to the electron momentum deviation $g$ and has the same sign. Consequently, in the case of negative $g$ we have the negative local potential of the edge site that finally causes the appearance of the edge state.

\section{Conclusions}

We presented the method for considering the edge state spectrum branch in the region where it splits from the band of extended states. The method is based on making use of a small parameter: the deviation of electron energy from the splitting point as compared with the energy gap. Due to this small parameter two characteristic regions of different (fast and slow) electron wave function behaviour appear where the simplified equations can be considered by analytic means. The method is demonstrated considering two toy models: the continuous version of the Bernevig model of a topological insulator and the discrete tight binding Haldane model of graphene.

In the case of both these models we managed to show that it is possible to convert the fast part equation into the boundary condition for the slow wave function part equation that finally determines the appearance of the edge state as an interplay of microscopic peculiarities of electron motion in the primitive cell with the mean electron motion along the edge. We also showed that the role of these boundary conditions can be interpreted as the appearance of the local potential at the edge asymmetric in respect to the electron momentum deviation from its momentum corresponding to the splitting point. Those splitting points do not coincide with the minima of continuous bands.

\section{Acknowledgements}

I thank E. Anisimovas for helpful discussions. This work was financially supported by the European Social Fund under the Global Grant Measure (Grant No. VP1-3.1-ŠMM-07-K-02-046).

\section{Appendix A}

\section{Single equation for continuous model}

We replace the two equation set (10) for components $u(x)$ and $v(x)$ by a single equation for the wave function component $u(x)$. For this purpose we multiply Eq. (10aa) by operator $\left(E+M-q^{2}+\mathrm{d}^{2} / \mathrm{d} x^{2}\right)$ and making use of Eq. (10b) rewrite it in the following way:

$$
\begin{aligned}
& \left(E+M-q^{2}+\frac{\mathrm{d}^{2}}{\mathrm{~d} x^{2}}\right)\left(E-M+q^{2}-\frac{\mathrm{d}^{2}}{\mathrm{~d} x^{2}}\right) u \\
& =\left[E^{2}-\left(M-q^{2}+\frac{\mathrm{d}^{2}}{\mathrm{~d} x^{2}}\right)^{2}\right] u \\
& =-\mathrm{i}\left(\frac{\mathrm{d}}{\mathrm{d} x}+q\right)\left(E+M-q^{2}+\frac{\mathrm{d}^{2}}{\mathrm{~d} x^{2}}\right) v \\
& =-\left(\frac{\mathrm{d}}{\mathrm{d} x}+q\right)\left(\frac{\mathrm{d}}{\mathrm{d} x}-q\right) u=\left(q^{2}-\frac{\mathrm{d}^{2}}{\mathrm{~d} x^{2}}\right) u
\end{aligned}
$$

So, we obtained the differential equation of the fourth order for a single component $u(x)$ that can be easily rewritten in the final form as Eq. (13).

The boundary conditions have to be reconsidered as well. Condition (11a) remains the same. But the second one, given by Eq. (11b), has to be replaced by the proper condition for the $u(x)$ component. For this purpose we multiply Eq. (10a) by the operator $\mathrm{i}(q-\mathrm{d} / \mathrm{d} x)$ and rewrite it as follows:

$$
\begin{aligned}
& \mathrm{i}\left(q-\frac{\mathrm{d}}{\mathrm{d} x}\right)\left(E-M+q^{2}-\frac{\mathrm{d}^{2}}{\mathrm{~d} x^{2}}\right) u \\
& =\left(q-\frac{\mathrm{d}}{\mathrm{d} x}\right)\left(q+\frac{\mathrm{d}}{\mathrm{d} x}\right) v=\left(q^{2}-\frac{\mathrm{d}^{2}}{\mathrm{~d} x^{2}}\right) v .
\end{aligned}
$$

The right hand side of the obtained equation can be found by resolving Eq. (10b):

$$
\left(q^{2}-\frac{\mathrm{d}^{2}}{\mathrm{~d} x^{2}}\right) v=\mathrm{i}\left(\frac{\mathrm{d}}{\mathrm{d} x}-q\right) u+(E+M) v .
$$


Comparing the obtained equation with Eq. (A2) we see that the component $u(x)$ has to satisfy the following equation as well:

$$
\begin{aligned}
& \mathrm{i}\left(q-\frac{\mathrm{d}}{\mathrm{d} x}\right)\left(E-M+1+q^{2}-\frac{\mathrm{d}^{2}}{\mathrm{~d} x^{2}}\right) u \\
& =(E+M) v .
\end{aligned}
$$

Now inserting $x=0$ into the obtained equation and taking formula (11b) into account we obtain the second final boundary condition (16b) for the $u(x)$ component.

\section{Appendix B}

\section{Single equation for TBM}

In order to convert the set of two differential equations (39) into a single equation for the component $u_{n}$ we calculate the sum of three equations: Eq. (39a) for $u_{n}$ multiplied by $(E-\tau \lambda)$, and the same equations for $u_{n \pm 1}$ multiplied by $\tau$. Then transforming the obtained result with the aid of Eq. (39b) we arrive at the following equation that we are looking for:

$$
\begin{aligned}
& \left(E^{2}-\lambda^{2} \tau^{2}-2 \tau^{2}-\lambda^{2}-1\right) u_{n} \\
& -\lambda\left(1-2 \tau^{2}\right)\left(u_{n-1}+u_{n+1}\right) \\
& -\tau^{2}\left(u_{n-2}+u_{n+2}\right)=0 .
\end{aligned}
$$

This procedure is correct for any $n \geq 2$. Performing the same procedure in the case of $n=1$ we have the term $\tau^{2} u_{-1}$ missing. However, this fact does not break the validity of the obtained equation (B1) if the boundary condition (41a) is taken into account.

We also need to replace the boundary condition (41b) by some condition for the component $u_{n}$. For this purpose we repeat the above procedure in the case of $n=0$. So, let us multiply Eq. (39a) for $n=0$ by $(E-\tau \lambda)$ and add to it the same equation for $n=1$ multiplied by $\tau$. In this case making use of Eq. (39b) for $n=0$ we have the following result:

$$
\begin{aligned}
& \left(E^{2}-\lambda^{2} \tau^{2}-\tau^{2}-\lambda^{2}\right) u_{0} \\
& -\lambda\left(1-2 \tau^{2}\right) u_{1}-\tau^{2} u_{2}=\tau v_{0} .
\end{aligned}
$$

In order to use this equation as the second boundary condition for the amplitude $u_{n}$ we have to eliminate the term containing the $v_{0}$ amplitude in the right hand side of that equation. This can be achieved writing down Eq. (39a) for $n=0$ and taking into account the boundary condition $(41 \mathrm{~b})$ what gives us the following equation:

$$
\begin{aligned}
& {\left[\lambda\left(E^{2}-\lambda^{2} \tau^{2}-\tau^{2}-\lambda^{2}\right)-\lambda \tau^{2}-\tau E\right] u_{0}} \\
& -\left[\lambda^{2}\left(1-2 \tau^{2}\right)-\tau^{2}\right] u_{1}-\lambda \tau^{2} u_{2}=0 .
\end{aligned}
$$

This equation can be simplified combining it with Eq. (B1) for $n=0$ that has to be satisfied as well. Superposition of these two equations enables us to present the secondary boundary condition in the following form:

$$
(\lambda-\tau E) u_{0}+\tau^{2} u_{1}+\lambda \tau^{2} u_{-2}=0
$$

Thus, Eq. (B1), the boundary condition (41a) and Eq. (B4) make the complete set of equations for a single amplitude $u_{n}$ that we were looking for and are going to use for the description of the edge state in the vicinity of the point where it splits off from the continuous spectrum. In order to be closer to the equations used in Section 2 when considering the continuous model of the topological insulator we regroup the terms of the above equations and rewrite them in the form that is given by the final Eqs. (46).

\section{References}

[1] M.Z. Hasan and C.L. Kane, Colloquium: Topological insulators, Rev. Mod. Phys. 82, 3045 (2010).

[2] K.S. Novoselov, A.K. Geim, S.V. Morozov, D. Jiang, Y. Zhang, S.V. Dubonos, I.V. Grigorieva, and A.A. Firsov, Electric field effect in atomically thin carbon films, Science 306, 666 (2004).

[3] C.L. Kane and E.J. Mele, Quantum spin Hall effect in graphene, Phys. Rev. Lett. 95, 226801 (2005).

[4] M.V. Berry, Quantal phase factors accompanying adiabatic changes, Proc. R. Soc. Lond. A 392, 45 (1984).

[5] J.E. Avron, D. Osadchy, and R. Seiler, A topological look at the quantum Hall effect, Physics Today 56, 38 (2003).

[6] D. Xiao, M. Chang, and Q. Niu, Berry phase effects on electronic properties, Rev. Mod. Phys. 82, 1959 (2010).

[7] D.J. Thouless, M. Kohmoto, M.P. Nightingale, and M. den Nijs, Quantized Hall conductance in a twodimensional periodic potential, Phys. Rev. Lett. 49, 405 (1982).

[8] X.G. Wen, Topological orders and edge excitations in fractional quantum Hall states, Adv. Phys. 44, 405 (1995).

[9] K. Wakabayashi, K.-I. Sasaki, T. Nakanishi, and T. Enoki, Electronic states of graphene nanoribbons and analytical solutions, Sci. Technol. 11, 054504 (2010). 
[10] S. Mao, Y. Kuramoto, K.-I. Imura, and A. Yamakage, Analytic theory of edge modes in topological insulators, J. Phys. Soc. Jpn. 79, 124709 (2010).

[11]V. Gurarie, Single-particle Green's functions and interacting topological insulators, Phys. Rev. B 83, 085426 (2011).

[12]E.B. Sonin, Edge accumulation and currents of moment in 2D topological insulators, Phys. Rev. B 82, 113307 (2010).

[13]B.A. Bernevig, T.L. Hughes, and S.C. Zhang, Quantum spin Hall effect and transition in $\mathrm{HgTe}$ wells, Science 314, 1757 (2006).
[14]S.V. Morozov, K.S. Novoselov, M.I. Katsnelson, F. Schedin, L.A. Ponomorenko, D. Jiang, and A.K. Geim, Strong suppression of weak localization in graphene, Phys. Rev. Lett. 97, 016801 (2006).

[15] H. Min, J.E. Hill, N.A. Sinitsyn, B.R. Sahu, L. Kleiman, and A.H. MacDonald, Intrinsic and Rashba spin-orbit interactions in graphene sheets, Phys. Rev. B 74, 165310 (2006).

[16]Y. Yao, F. Ye, X.-L. Qi, S.-C. Zhang, and Z. Fang, Spin-orbit gap of graphene: First-principles calculations, Phys. Rev. B 75, 041401 (2007).

\title{
DVISKALĖ KRAŠTINĖS BŪSENOS TEORIJA
}

\author{
A. Matulis \\ Fizinių ir technologijų mokslų centro Puslaidininkių fizikos institutas, Vilnius, Lietuva
}

\section{Santrauka}

Išnagrinèta kraštinė būsena spektro srityje, kur jos energijos šaka atskyla nuo tolydinès laidumo juostos. Parodyta, kad šioje srityje elektrono banginè funkcija demonstruoja charakteringą dviejų skirtingų mastelių elgesį: greitą ir lètą kitimą koordinatei tolstant nuo krašto. Tai igalino sukonstruoti tam tikrą paprastą kraštinès būsenos analizès procedūrą panaudojant mažą parametrą - energijos nuokrypio nuo jos ịsiliejimo ị tolydinę juostą taško ir draustinès juostos energijos santyki. Lèto banginès funkcijos kitimo srityje ji tenkina paprastą Šrèdingerio lygtị, kurios parametrai nejautrūs detaliai elektrono dinamikai primityviajame narvelyje. Ta detali dinamika pasireiškia tik greitojo funkcijos kitimo srityje, kuri sukoncentruota prie paties krašto. Lygti šioje greitojo kitimo srityje pasisekè transformuoti j kraštinę sąlygą lètojo funkcijos kitimo lygčiai, taip suformuluojant dviskalę kraštinès būsenos teoriją. Metodas iliustruotas ji pritaikant papraščiausiam tolydiniam topologinio izoliatoriaus modeliui ir stipraus ryšio grafeno modeliui, kuriame iskaityti sukinio-orbitos sąveika ir elektrono tuneliavimas ị tolesnius kaimyninius mazgus. 